\title{
Superscaling in electron- and neutrino-nucleus scattering
}

\author{
Maria B. Barbaro \\ Dipartimento di Fisica Teorica, Università di Torino and INFN, Sezione di Torino, Italy
}

The superscaling properties of electron scattering data are used to extract model-independent predictions for neutrino-nucleus cross sections.

\section{Introduction}

The analyses of many of the ongoing and future neutrino experiments require reliable predictions of the neutrino-nucleus cross sections. Various model calculations have been performed in the quasielastic peak (QEP) region, where the dominant process is the knock-out of a single nucleon. However, the uncertainty due to different treatments of the nuclear dynamics is still high when compared with the required precision.

The discrepancy between various models is even more pronounced when one moves from the QEP to higher energy transfers, where the nucleonic resonances, in particular the Delta, come into play. A complete description of the so-called "dip" region between the $\mathrm{QE}$ and the $\Delta$ peak is in fact still missing.

Of course any nuclear model to be applied to neutrino scattering should first be tested against electron scattering data, where a much higher statistics exists than in neutrino-nucleus physics which allows to discriminate between different models. It is well-known that the simple Fermi gas (FG) model, although accounting for the gross features of the inclusive $\left(e, e^{\prime}\right)$ differential cross sections, is inadequate for a detailed description of lepton-nucleus reactions. Specifically, the FG overestimates the ratio between the longitudinal and transverse responses at the QEP and predicts sharp edges of the cross section versus the energy transfer $\omega$, in contrast with the tails displayed by the experimental data. Moreover, it predicts a linear behavior of the cross sections at low $\omega$ and $q$ which is not supported by the experiment.

For this reason more sophisticated models and techniques (including RPA, final state interactions, short range correlations, Green's function methods) have been used in the last decades to describe electron scattering reactions. Most of these models are based on a non-relativistic treatment of the nuclear problem, which indeed works quite well in the low and intermediate domain of momentum transfers up to about $500 \mathrm{MeV} / \mathrm{c}$. However, in the energy regime of few $\mathrm{GeV}$ of interest for modern neutrino experiments, relativistic effects cannot be ignored.

Although several efforts to account for relativistic effects in neutrino scattering have recently been made (see, e.g., Refs. 2234]), a consistent relativistic description of lepton-nucleus cross sections is hard to achieve [5].

To overcome this difficulty, an alternative approach can be taken 6]: instead of using a specific nuclear model, one can try to extract modelindependent predictions for $\nu-A$ from $e-A$ experimental cross sections.

The method relies on the superscaling properties of the electron scattering data: at sufficiently high momentum transfer the inclusive differential $\left(e, e^{\prime}\right)$ cross sections, divided by a suitable function which takes into account the single nucleon content of the problem, depends only upon one kinematical variable, the scaling variable (this behavior is called scaling of first kind) and the resulting function is roughly the same for all nuclei (scaling of second kind). When both kinds of scaling are fulfilled the cross section is said to superscale.

More specifically the scaling function

$f=\frac{d^{2} \sigma / d \Omega d k^{\prime}}{S(q, \omega)}$ 
being $S$ related to the single nucleon cross section (see [6]), becomes, for large $q$, a function only of the scaling variable

$\psi_{\mathrm{QE}}= \pm \sqrt{1 /\left(2 T_{F}\right)(q \sqrt{1+1 / \tau}-\omega-1)}$,

where $T_{F}$ is the Fermi kinetic energy, $4 m_{N}^{2} \tau=$ $q^{2}-\omega^{2}$ and the $-(+)$ sign corresponds to energy transfers lower (higher) than the QEP $(\psi=0)$.

An extensive analysis of electron scattering data [7] has shown that scaling of the first kind is fulfilled at the left of the QEP and broken at its right, whereas scaling of the second kind is very well satisfied at the left side of the peak and not so badly violated at its right. As a consequence a scaling function $f^{\mathrm{QE}}\left(\psi_{\mathrm{QE}}\right)$, which embodies the scaling part of the nuclear effects for any momentum transfer $q$ and any nucleus (provided $q$ is high enough, namely larger or of the order of about 400 $\mathrm{MeV} / \mathrm{c}$ ) can be extracted from the data.

The scaling violations observed at high energy transfers reside in the transverse response function and their main origin is the excitation of a $\Delta$ resonance.

The superscaling analysis has been extended to the first resonance peak in Ref. [6], where the contribution of the $\Delta$ has been (approximately) isolated in the experimental data by subtracting the quasielastic scaling contribution from the total experimental cross sections; the reduced cross section has been studied as a function of a new scaling variable

$\psi_{\Delta}= \pm \sqrt{1 /\left(2 T_{F}\right)(q \sqrt{\rho+1 / \tau}-\omega \rho-1)}$,

which accounts for the inelasticity through the quantity $\rho=1+\left(m_{\Delta}^{2}-m_{N}^{2}\right) /\left(4 \tau m_{N}^{2}\right)$. The results show that also in this region superscaling is working quite well and therefore a second superscaling function, $f^{\Delta}\left(\psi_{\Delta}\right)$, can be extracted from the data to account for the nuclear dynamics. Clearly this approach can work only at $\psi_{\Delta}<0$, since at $\psi_{\Delta}>0$ other resonances and the tail of the deep-inelastic scattering start contributing.

The two scaling functions extracted from $\left(e, e^{\prime}\right)$ data can then be used to predict neutrino cross sections in the same kinematical range. Before showing the results, we briefly summarize the formalism for neutrino-nucleus scattering.

\section{Formalism}

\subsection{Charged-current reactions}

The neutrino-nucleus cross section can be written in terms of response functions in a generalized Rosenbluth decomposition $(q \| z)$. For chargechanging processes

$$
\nu+A \rightarrow l+B
$$

this reads $\underline{6}$

$$
\begin{aligned}
& {\left[\frac{d^{2} \sigma}{d \Omega d k^{\prime}}\right]_{\chi}=\sigma_{0}^{(C C)}\left\{\widehat{V}_{C C} R_{C C}+2 \widehat{V}_{C L} R_{C L}\right.} \\
& \left.+\widehat{V}_{L L} R_{L L}+\widehat{V}_{T} R_{T}+2 \chi \widehat{V}_{T^{\prime}} R_{T^{\prime}}\right\}
\end{aligned}
$$

where $\Omega$ and $k^{\prime}$ are the scattering angle and momentum of the outgoing lepton. In (4) $\sigma_{0}$ and $\widehat{V}$ are kinematical factors and $\chi=+1(-1)$ for neutrino (antineutrino) scattering.

In the Relativistic Fermi Gas (RFG) model the nucleus is viewed as a collection of on-shell nucleons moving with energy $E_{p}=\sqrt{p^{2}+m_{N}^{2}}$ and described by free Dirac spinors $u(\mathbf{p}, s)$. In spite of its simplicity the model fulfills two fundamental requirements which are usually difficult to satisfy: Lorentz covariance (the hadronic tensor transforms as a Lorentz tensor) and gauge invariance (the nuclear vector current is conserved). Moreover it allows for analytic expressions of the response functions:

$R_{R F G}^{(i)}(q, \omega)=\mathcal{N} \frac{m_{N} T_{F}\left|Q^{2}\right|}{k_{F}^{3} q} R_{s . n .}^{(i)}(q, \omega) f_{R F G}(\psi)$,

where $\mathcal{N}=Z, N$ is the nucleon number, $k_{F}$ the Fermi momentum and $R_{s . n}^{(i)}$. the "single nucleon" response corresponding to the elementary reaction. The function

$f_{R F G}(\psi)=\frac{3}{4}\left(1-\psi^{2}\right) \theta\left(1-\psi^{2}\right)$

is universal: when plotted against the scaling variable $\psi\left(q, \omega ; k_{F}\right)$ no dependence is left either on the momentum transfer $q$ or on the nuclear species via $k_{F}$. As above mentioned, we then say 
that the RFG superscales. Note that the RFG response region is restricted to $-1 \leq \psi \leq 1$.

The above expressions hold for both the QE and the $\Delta$ peaks, the only differences being the single nucleon tensor (which corresponds to the elementary reaction $\nu N \rightarrow l N$ and $\nu N \rightarrow l \Delta$, respectively) and the scaling variable, which is different because of the different kinematics. In particular the QE and $\Delta$ peaks correspond to $\psi=$ 0 , which implies $\omega^{P}=\left|Q^{2}\right| /\left(2 m_{N}\right)$ for $\mathrm{QE}$ and $\omega^{P}=\left(\left|Q^{2}\right|+\left(m_{\Delta}^{2}-m_{N}^{2}\right)\right) /\left(2 m_{N}\right)$ for the $\Delta$ peak.

The RFG model approximately reproduces the shape and position of the two peaks, providing an energy shift, which accounts for the nucleon separation energy, is introduced in the model through the substitution $\omega \rightarrow \omega-E_{\text {shift }}$, entailing $\psi \rightarrow \psi^{\prime}$. Note that $E_{\text {shift }}$, typically of the order of $20 \mathrm{MeV}$, and the Fermi momentum $k_{F}$ are the only parameters of the model.

To go beyond the RFG according to the procedure illustrated in the previous section, we just replace the superscaling function $f_{R F G}$ with $f^{\mathrm{QE}}$ and $f^{\Delta}$, extracted directly from the experiment in the $\mathrm{QE}$ and $\Delta$ regions, respectively.

\subsection{Neutral-current reactions}

For neutral current (NC) processes

$$
\nu+A \rightarrow \nu^{\prime}+(A-1)+N
$$

an expression similar to (4) holds [8]:

$$
\begin{aligned}
& {\left[\frac{d^{2} \sigma}{d \Omega_{N} d p_{N}}\right]_{\chi}=\sigma_{0}^{(N C)}\left\{V_{L} R_{L}+V_{T} R_{T}\right.} \\
& +V_{T T} R_{T T}+V_{T L} R_{T L} \\
& \left.+2 \chi\left(V_{T^{\prime}} R_{T^{\prime}}+V_{T L^{\prime}} R_{T L^{\prime}}\right)\right\},
\end{aligned}
$$

where $\Omega_{N}$ and $p_{N}$ are the scattering angle and momentum of the emitted nucleon and now 6 response functions appear.

In this case the final state which is detected is the outgoing nucleon, and the neutrino kinematic variables are integrated over. Therefore the kinematics of the reaction is different from the one of $\mathrm{CC}$ processes and of $\left(e, e^{\prime}\right)$ : these are $t$-scattering type processes (the Mandelstam variable $t$ is fixed), whereas the neutral current reactions are $u$-scattering processes (the variable
$u=k-p_{N}$ is fixed and $t$ is integrated over) 9]. As a consequence, it is not obvious that the superscaling procedure, based on the analogy with inclusive electron scattering, is still valid.

This issue is discussed at length in Ref. 8, where it is shown that the scaling method is based on a factorization assumption which has to be tested numerically. Actually, as illustrated in [8], the scaling method appears to be applicable also to neutral current reactions, at least in the QEP region. The resonance production would be more involved, since the final state involves both a nucleon and a pion.

\section{Results}

Before showing the results for neutrino reactions we test the validity of the function $f^{\mathrm{QE}}\left(\psi_{\mathrm{QE}}\right)+f^{\Delta}\left(\psi_{\Delta}\right)$ as a representation of the nuclear dynamics for electron scattering in the quasielastic and $\Delta$ peaks. In Fig. 1 the cross section reconstructed by multiplying the phenomenological superscaling function by the appropriate single nucleon functions is compared with the data for different kinematics and different nuclei. It appears that typical deviations are $10 \%$ or less, thus confirming that the scaling approach offers a reliable description of the nuclear dynamics.

Turning now to neutrino scattering, our prediction for the CC process is shown in Fig.2 for scattering of $1 \mathrm{GeV}$ neutrinos off carbon at $\theta=45^{\circ}$ as a function of the outgoing muon momentum $k^{\prime}$. The full result obtained using the empirical scaling functions $f^{\mathrm{QE}}$ and $f^{\Delta}$ is compared with the result obtained using the RFG model $f_{R F G}$. It appears that the RFG cross section differs significantly from the scaling prediction, which lies somewhat lower and extends over a wider range in $k^{\prime}$. More results are shown in Ref. 6, for different kinematics and including antineutrino scattering. Here we just summarize the main findings. Concerning the relative weight of the $\mathrm{QE}$ and $\Delta$ contributions, we find that at low angles the QE contribution dominates over the one coming from the $\Delta$, the two contributions becoming comparable for $\theta \sim 90^{\circ}$. Moreover, a large difference between $\nu$ and $\bar{\nu}$ cross sections is predicted 
at backward angles due to the balance between the various contributions to the total response (see Eq. (4)). For both the $\nu$ and $\bar{\nu}$ cases the transverse and axial-transverse responses $R_{T}$ and $R_{T^{\prime}}$ are the dominant ones, whereas the contributions of $R_{C C}, R_{C L}$ and $R_{L L}$ is less than $5 \%$. However $\widehat{V}_{T} R_{T} \simeq \widehat{V}_{T^{\prime}} R_{T^{\prime}}$, so that they interfere constructively in $\nu$ scattering, but tend to cancel in $\bar{\nu}$ scattering. As a consequence small changes in the nuclear model could have large effects on the antineutrino cross sections.

Concerning the neutral current reactions, we show in Fig. 3 (upper panel) the comparison between the RFG and the superscaling results in the quasi-elastic peak. As in the CC case, the empirical superscaling function predicts a lower and more extended differential cross section.

It is well-known (see, e.g., Refs. [1110]) that the $\mathrm{NC}$ reactions are very sensitive to the strangeness content of the nucleon and are then used, together with parity-violating electron scattering [1112, to measure the strange form factors of the nucleon. We show in Fig.3 (lower panel) the cross section obtained from the phenomenological superscaling function in a situation where no strangeness is assumed (solid line) with the ones obtained including strangeness in the magnetic (long-dashed) and axial-vector (dotted) form factors, using for $\mu_{s}=G_{M}^{(s)}(0)$ a representative value extracted from the recent world studies of parity-violating electron scattering and taking $g_{A}^{s}=G_{A}^{(s)}(0)$ to be -0.2 . The effects from inclusion of electric strangeness are not shown here, since $G_{E}^{(s)}$ has almost no influence on the full cross sections.

\section{Conclusions}

We have illustrated a method which yields predictions for neutrino-nucleus cross sections by extracting the scaling function from electron scattering data in the kinematical regions of the quasi-elastic peak and of the $\Delta$-resonance. The method reproduces the inclusive $\left(e, e^{\prime}\right)$ data in a wide range of kinematical conditions and for different nuclear targets. It has the merit of being model independent and accounts for that part of the nuclear dynamics which superscales.
The cross sections obtained by using this procedure are sensibly different from the ones obtained in the relativistic Femi gas model, which is therefore questionable when used in analyses of neutrino experiments.

The error implicit in this procedure is related to the violations of superscaling. These mainly arise from meson-exchange currents and their associated correlations in both the particle-hole [13. 1415] and the two particle-two hole [1617 sectors, whose impact is bound by the data to be within at most $10 \%$.

Of course the specific nature of the scaling function should be accounted for by any reliable microscopic calculation. In particular the origin of the asymmetric shape of $f^{\mathrm{QE}}$ with respect to the scaling variable is hard to be explained on a microscopic basis and represents a valid test of different nuclear models [4].

\section{Acknowledgments}

The work presented has been carried out in collaboration with J.E. Amaro, J.A. Caballero, T.W. Donnelly, A. Molinari and I. Sick.

\section{REFERENCES}

1. see contributions of O. Benhar and G. P. Co', this conference.

2. A. Meucci, C. Giusti and F. D. Pacati, Nucl. Phys. A 739 (2004) 277.

3. J. E. Amaro, M. B. Barbaro, J. A. Caballero, T. W. Donnelly and C. Maieron, Phys. Rev. C 71 (2005) 065501.

4. J. A. Caballero, J. E. Amaro, M. B. Barbaro, T. W. Donnelly, C. Maieron and J. M. Udias, Phys. Rev. Lett. 95 (2005) 252502.

5. J. E. Amaro, M. B. Barbaro, J. A. Caballero, T. W. Donnelly and A. Molinari, Phys. Rept. 368 (2002) 317.

6. J. E. Amaro, M. B. Barbaro, J. A. Caballero, T. W. Donnelly, A. Molinari and I. Sick, Phys. Rev. C 71 (2005) 015501.

7. C. Maieron, T. W. Donnelly and I. Sick, Phys. Rev. C 65 (2002) 025502; T. W. Donnelly and I. Sick, Phys. Rev. C 60 (1999) 065502; 
T. W. Donnelly and I. Sick, Phys. Rev. Lett. 82 (1999) 3212.

8. J. E. Amaro, M. B. Barbaro, J. A. Caballero and T. W. Donnelly, submitted to Phys. Rev. C.

9. M. B. Barbaro, A. De Pace, T. W. Donnelly, A. Molinari and M. J. Musolf, Phys. Rev. C 54 (1996) 1954.

10. W. M. Alberico et al., Nucl. Phys. A 623 (1997) 471.

11. M. J. Musolf, T. W. Donnelly, J. Dubach, S. J. .. Pollock, S. Kowalski and E. J. Beise, Phys. Rept. 239 (1994) 1.

12. T. W. Donnelly, M. J. Musolf, W. M. Alberico, M. B. Barbaro, A. De Pace and A. Molinari, Nucl. Phys. A 541, 525 (1992).

13. J. E. Amaro, M. B. Barbaro, J. A. Caballero, T. W. Donnelly and A. Molinari, Nucl. Phys. A 723, 181 (2003) arXiv:nucl-th/0301023.

14. J. E. Amaro, M. B. Barbaro, J. A. Caballero, T. W. Donnelly and A. Molinari, Nucl. Phys. A 697, 388 (2002) arXiv:nucl-th/0106035.

15. J. E. Amaro, M. B. Barbaro, J. A. Caballero, T. W. Donnelly and A. Molinari, Nucl. Phys. A 643, 349 (1998) arXiv:nucl-th/9806014.

16. A. De Pace, M. Nardi, W. M. Alberico, T. W. Donnelly and A. Molinari, Nucl. Phys. A 741, 249 (2004) arXiv:nucl-th/0403023.

17. A. De Pace, M. Nardi, W. M. Alberico, T. W. Donnelly and A. Molinari, Nucl. Phys. A 726, 303 (2003) arXiv:nucl-th/0304084.
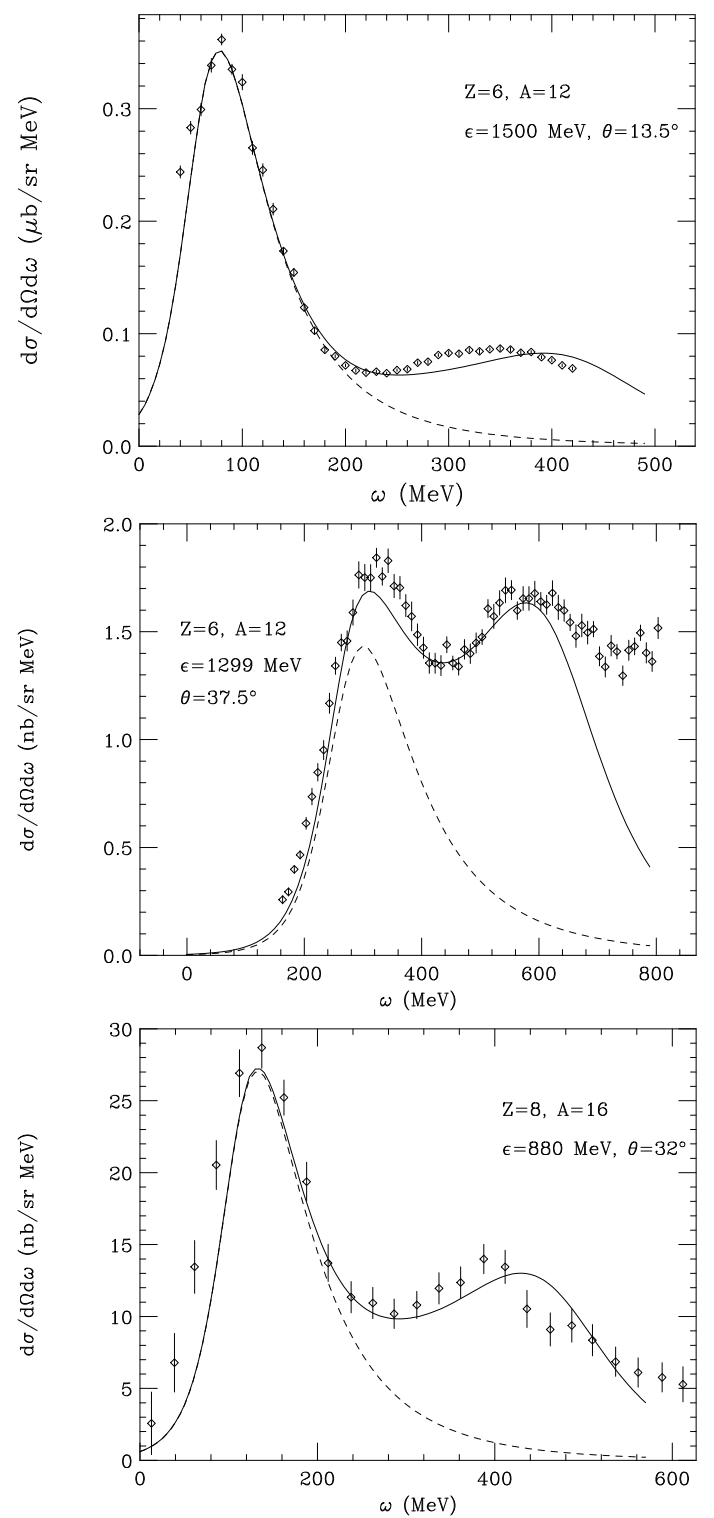

Figure 1. Experimental $\left(e, e^{\prime}\right)$ cross section for ${ }^{12} \mathrm{C}$ and ${ }^{16} \mathrm{O}$ at different incident electron energies and scattering angles. The solid curves represent the calculated result obtained using $f^{\mathrm{QE}}$ and $f^{\Delta}$, whereas the dashed curves are the QE contributions. (Ref. [6]) 


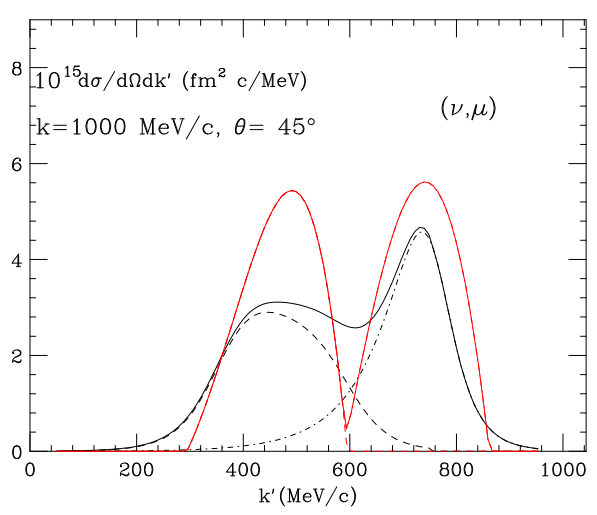

Figure 2. Charge-changing neutrino cross section for for $1 \mathrm{GeV}$ neutrinos on ${ }^{12} \mathrm{C}$ and neutrinomuon scattering angles of 45 degrees. The cross section is plotted versus the final-state muon momentum $k^{\prime}$. Solid line: superscaling prediction; heavier line (red on line): RFG. The separate $\mathrm{QE}$ and $\Delta$ contribution are shown (dotted lines). (Ref. [6])

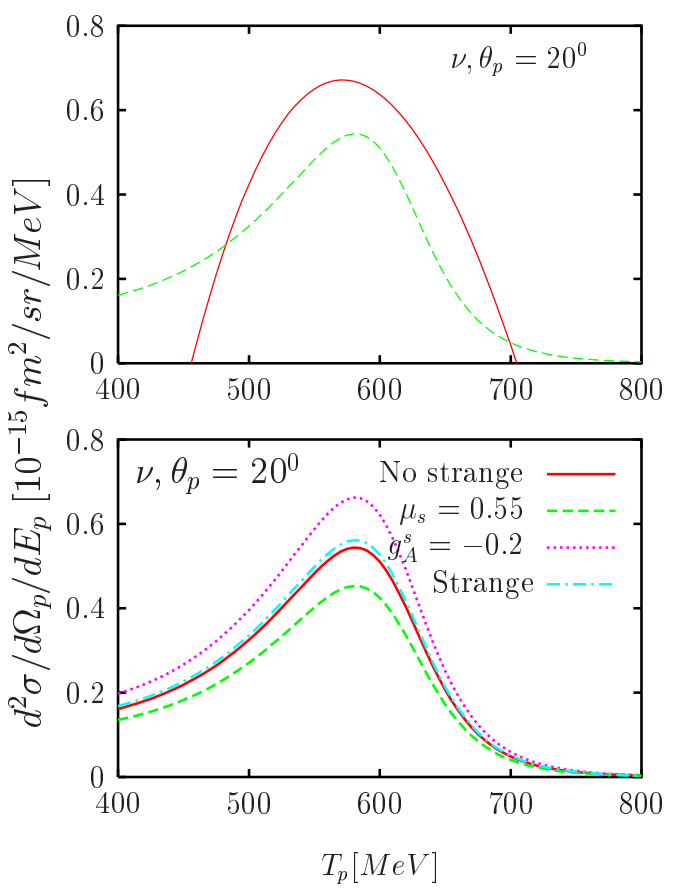

Figure 3. Upper panel: quasielastic differential cross section for neutral current neutrino scattering at $1 \mathrm{GeV}$ from ${ }^{12} \mathrm{C}$ for proton knockout obtained using the RFG (solid), and the empirical scaling function (dashed). $T_{p}$ is the outgoing proton kinetic energy. Lower panel: sensitivity to the magnetic and axial strangeness content of the nucleus. (Ref. [8]) 